\title{
Non-volant mammal inventory of Cusuco National Park, north- west Honduras: reporting the presence of Jaguar, Panthera onca (Linnaeus, 1758), and demonstrating the effects of zonal protection on mammalian abundance
}

\author{
Hannah M.J. Hoskins ${ }^{1,2}$, Oliver J. Burdekin ${ }^{2,3}$, Kara Dicks ${ }^{1}$, Kathy Y. Slater ${ }^{2}$, Niall P. McCann², \\ Merlijn Jocque ${ }^{2,4}$, Franklin Castañeda ${ }^{5}$, Neil Reid ${ }^{1,2,6}$
}

1 School of Biological Sciences, Queen's University Belfast, MBC, 97 Lisburn Road, Belfast, BT9 7BL. 2 Operation Wallacea, Wallace House, Old Bolingbroke, Lincolnshire, PE23 4EX, UK. 3 burdGIS, Unit G37b, Market Walk, Wakefield, WF1 1QR, UK. 4 Royal Belgian Institute of Natural Sciences, Aquatic \& Terrestrial Ecology, Vautierstraat 29, 1000 Brussels, Belgium. 5 Panthera, 3415, Bloque L, Los Robles, Tegucigalpa, Honduras. 6 Institute of Global Food Security (IGFS), Queen's University Belfast, 18-30 Malone Road, Belfast, BT9 5BN.

Corresponding author: Hannah M.J. Hoskins, hhoskins01@qub.ac.uk

\begin{abstract}
Neotropical cloud forests are one of the most biodiverse yet threatened ecosystems with deforestation and hunting creating major problems. We report a non-volant mammal species inventory for Cusuco National Park, Honduras, spanning 2006-2016. Data were collected using 4 methods: tracks and signs, camera trapping, direct sightings and live trapping. Recorded species of conservation importance included Central American red brocket (Mazama temama (Kerr, 1792)), Baird's tapir (Tapirus bairdii (Gill, 1865)), margay (Leopardus wiedii (Schinz, 1821)), and jaguar (Panthera onca (Linnaeus, 1758)); the latter recorded for the first time. Cusuco National Park is a site of strategic geographical importance within the Central American jaguar corridor. In total, 43 species were recorded from 26 families. We demonstrate the impact of zonal protection measures with higher mammal abundance recorded in the park's highly protected core compared to the less protected buffer zone. We advise continued monitoring to provide robust time-series of population trends.
\end{abstract}

\section{Key words}

Bushmeat; diversity; Tapirus bairdii; brocket; Central America.

Academic editor: William Corrêa Tavares | Received 22 February 2018 | Accepted 11 July 2018 | Publised 19 October 2018

Citation: Hoskins HMJ, Burdekin OJ, Dicks K, Slater KY, McCann NP, Jocque M, Castañeda F, Reid N (2018) Non-volant mammal inventory of Cusuco National Park, north-west Honduras: reporting the presence of Jaguar, Panthera onca (Linnaeus, 1758), and demonstrating the effects of zonal protection on mammalian abundance. Check List 14 (5): 877-891. https://doi.org/10.15560/14.5.877

\section{Introduction}

Neotropical cloud forests form part of the Mesoamerican biodiversity hotspot located within the Panamanian biogeographical realm (Myers et al. 2000). Cloud forest is one of the most threatened ecosystems (Higuera et al. 2012, Rojas-Soto et al. 2012) due to human encroach- ment, deforestation, forest degradation (e.g. fuel wood collection), hunting (e.g. for bushmeat due to food insecurity, reducing family expenditure, sport or tradition), road construction, and global climate change (Foster 2001, Luna-Vega et al. 2001, Ponce-Reyes et al. 2013) 
compounded by altitudinal isolation (Rojas-Soto et al. 2012).

Neotropical cloud forests are typified by high altitude, high precipitation and regular immersion in cloud (Stadtmüller 1987, Scatena et al. 2010). Parque Nacional Cusuco (hereafter referred to as Cusuco National Park), in north-west Honduras, is a protected high altitude forest within which there are 4 principal habitats; i) tropical lowland dry forest, ii) tropical moist forest, iii) montane (cloud) forest and iv) 'bosque enano' or dwarf forest occurring above 2,000 $\mathrm{m}$ a.s.1. Originally granted protected status due to its importance as a water catchment (Mejía 2001), the park is recognized by the International Union for the Conservation of Nature (IUCN) as a Key Biodiversity Area (KBA) due to the overlapping ranges of several globally threatened endemic amphibians (BirdLife International 2017). Cusuco National Park is ranked as the $123 \mathrm{rd}$ most irreplaceable site, from over 173,000 protected areas, for the conservation of threatened amphibians, birds and mammals (Le Saout et al. 2013). Furthermore, jaguar (Panthera onca (Linnaeus 1758)); an IUCN Red-Listed Near Threatened species with a decreasing population trend (Quigley et al. 2017) has gone unrecorded in Cusuco National Park, which is considered to be an important and critical section of the Central American jaguar corridor (Petracca et al. 2017, Wultsch et al. 2016). Additionally, it has been identified as a regional hotspot and important connection node for Baird's tapir (Tapirus bairdii Gill 1865) (Schank et al. 2017), an IUCN Red-Listed Endangered species with a decreasing population trend (Garcìa et al. 2016).

Despite its designation, Cusuco National Park is threatened by illegal logging (particularly in the north and west) and agricultural encroachment due to coffee, cardamom, crop and grassland agriculture. Large mammals in the region are threatened by illegal hunting for bushmeat and sport. Baird's tapir has declined within the park as demonstrated by dramatically decreased encounter rates and apparent range contraction with VORTEX population viability modelling suggesting its imminent local extirpation (McCann et al. 2012). Despite previous research focusing on tapir, there is a paucity of published data on the mammalian community within the park. There is no published species inventory for the region.

Bushmeat hunting is prevalent within the park, yet conservation action can only be effective with accurate data capture and characterization of the biodiversity present and the species that are rare or threatened. Consequently, we report here a species list for Cusuco National Park with the frequency and type of species records.

\section{Methods}

Study area. Cusuco National Park $\left(15^{\circ} 32^{\prime} 31^{\prime \prime} \mathrm{N}, 088^{\circ}\right.$ $15^{\prime} 49^{\prime \prime} \mathrm{W}$ ), is 23,440 ha of protected area (ICF 2015) in Sierra de Omoa of the Merendón mountain range, Cortés, north-west Honduras (Fig. 1). The Sierra de Omoa are isolated by the alluvial Sula valley to the east and Río
Chamelecón valley to the south and southeast, the Río Motagua alluvial plain to the west and northwest and the Bahía de Omoa to the north. The park, a mountainous cloud forest region, rises to $2,240 \mathrm{~m}$ above sea level, annual precipitation is $2,788 \mathrm{~mm}$, with mean monthly temperatures ranging from $12.9{ }^{\circ} \mathrm{C}$ in December to $20.2{ }^{\circ} \mathrm{C}$ in April (Fundación Ecologista 1994). During 1994, the Corporación Hondureña de Desarrollo Forestal (COHDEFOR), delineated the management plan of the park into a 'core zone' covering 7,690 ha where agriculture, burning, mining, hunting, construction of roads, houses, or commercial, public and private institutions or any human settlements are not permitted (ICF 1987). This region was surrounded by a 'buffer zone' covering 15,750 ha within which construction of human settlements, excluding those that existed before the implementation of the management plan, are permitted only under license as well as limited livestock grazing, burning, deforestation, mining, fishing, housing and road construction. Subsistence hunting may be permitted in this area but only if a permit is obtained, which requires travelling to San Pedro Sula, the regional administrative capital (a minimum of 4 hours travel away), and requires applicants to be literate, have understanding of the relevant paper work and transport; consequently, bushmeat hunting is unlicensed and, therefore, illegal.

Since 2006, a total of 7 camps (recording stations) have been established throughout Cusuco National Park; Base camp $\left(15^{\circ} 29^{\prime} 47^{\prime \prime} \mathrm{N}, 088^{\circ} 12^{\prime} 43^{\prime \prime} \mathrm{W}\right)$, Cantiles $\left(15^{\circ} 30^{\prime} 48^{\prime \prime} \mathrm{N}\right.$,

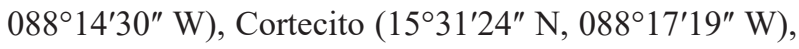
Danto $\left(15^{\circ} 31^{\prime} 42^{\prime \prime} \mathrm{N}, 088^{\circ} 16^{\prime} 39^{\prime \prime} \mathrm{W}\right)$, and Guanales (15 $\left.29^{\prime} 19^{\prime \prime} \mathrm{N}, 088^{\circ} 14^{\prime} 03^{\prime \prime} \mathrm{W}\right)$ in the core zone, and the villages of Buenos Aires (15 $\left.29^{\prime} 59^{\prime \prime} \mathrm{N}, 088^{\circ} 10^{\prime} 46^{\prime \prime} \mathrm{W}\right)$ and Santo Tomas $\left(15^{\circ} 33^{\prime} 42^{\prime \prime} \mathrm{N}, 088^{\circ} 18^{\prime} 01^{\prime \prime} \mathrm{W}\right)$ in the buffer zone. A transect network was established in 2006 by cutting (with machetes) 4 approximately $2 \mathrm{~km}$ line transects radiating out from each camp (approximately $8 \mathrm{~km}$ for each of the 7 camps with 28 transects and $56 \mathrm{~km}$ in total) along natural features including valley bottoms or ridges that could be relatively easily traversed. Species records for non-volant, terrestrial mammals were collected as part of long-term annual biodiversity monitoring and surveillance by a range of students, supervised by academics and local trackers using a range of methods.

Permits to carry out research were obtained from the Instituto Nacional de Conservación y Desarrollo Forestal Áreas Protegidas y Vida Silvestre (ICF), or National Institute for Conservation and Forestry Development, Protected Areas and Wildlife.

Data Collection. Monitoring and surveillance of small and large mammals was conducted annually during the decade 2006-2016 by Operation Wallacea Ltd (http://opwall.com) as part of their global biodiversity monitoring effort. Camera trap surveys were also conducted independently by Panthera (https://www.panthera.org/) during 3 years (20142016) as part of their Jaguar Corridor Initiative.

Small mammals were categorized as those $<2 \mathrm{~kg}$ in 


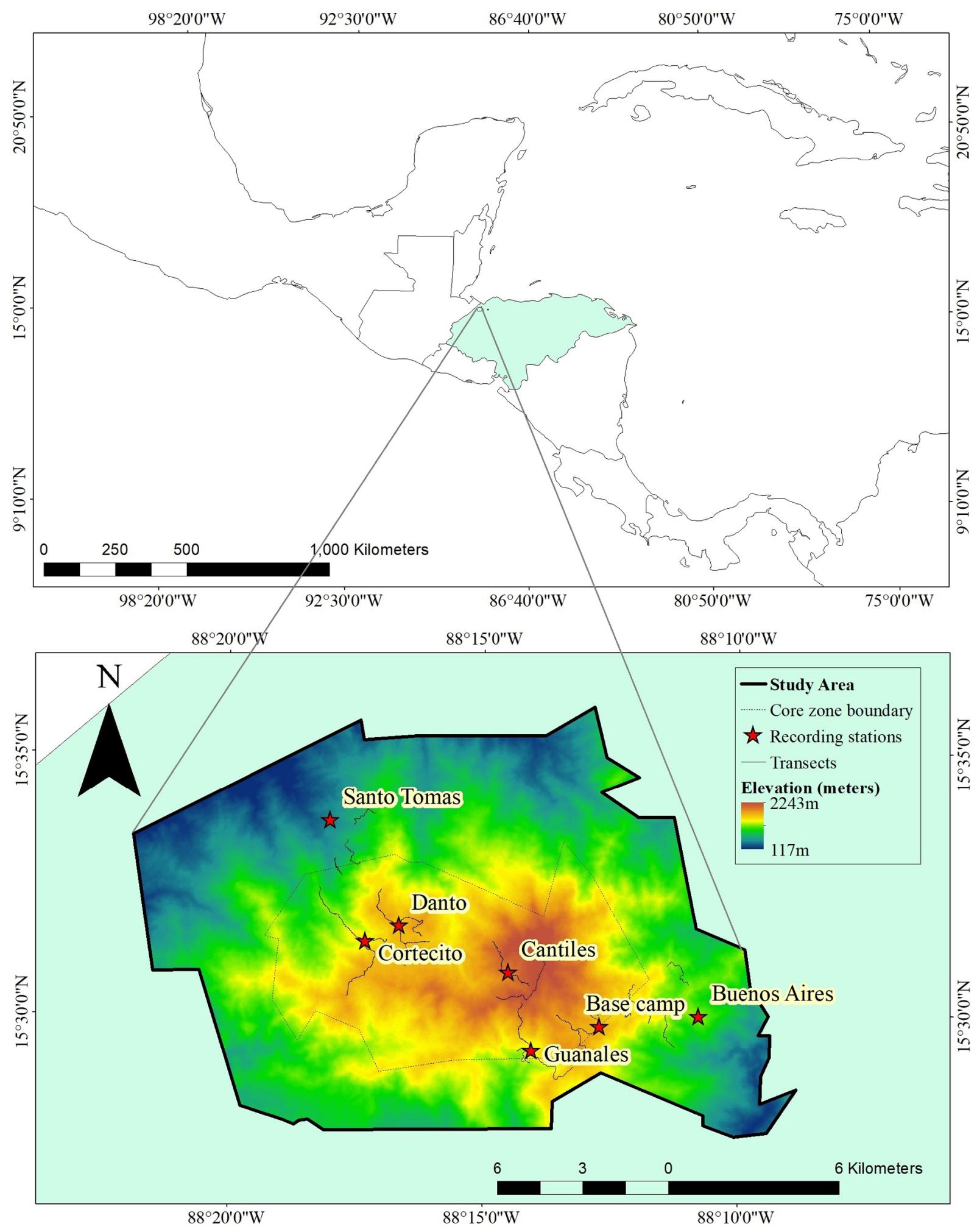

Figure 1. Cusuco National Park, North West Honduras.

body weight. A combination of 6 Freya $(320 \times 173 \times 140$ $\mathrm{mm})$ and 6 Sherman traps $(508 \times 635 \times 1651 \mathrm{~mm})$ were set in alternate pairs placed at $10 \mathrm{~m}$ intervals for $60 \mathrm{~m}$ for a period of 4 consecutive nights and checked daily at sunrise. Traps were baited alternately with a mixture of oats, peanut butter and syrup or tuna. Typically, 2 trap lines were set out on each transect with one placed $<5 \mathrm{~m}$ from and parallel to a stream or river (sampling the riparian corridor) and another placed $>150 \mathrm{~m}$ from the nearest watercourse (sampling the forest floor) (see Appendix for the list of camps surveyed each year). Surveys were conducted annually between June and August from 2006-2016, totaling 2,376 trap nights in the core zone and 1,272 trap nights in the buffer.

Captures were identified by means of gross external morphology; usually a combination of coloration (including presence of countershading, bicoloration of the tail, etc.), shape and head-body length, tail length, 
hind foot length and body weight following Reid (1997, 2009) and Wilson et al. (2017). For the majority, speciesspecific identification was not contentious as most were well described and commonly found species. However, 2 genera presented particular issues: Handleyomys and Cryptotis, which have undergone recent taxonomic revision with the description of new cryptic species (e.g. Woodman 2011, 2015, Almendra et al. 2018). Thus, notwithstanding cryptic species, we used gross morphological characters to differentiate captures to species level but, when reporting here, we adopt the use of "cf." to suggest a specimen's species membership.

Cryptotis presented particular problems as many shrew species are indistinguishable by external morphology, with a number of cryptic species being described recently (Woodman 2011, 2015). Thus, a small number of voucher specimens were taken by lethal sampling and lodged with the Royal Museum of Scotland, Edinburgh. Tissue samples were extracted from 10 individuals (2 of which were long-tailed with a TL $=50-53 \mathrm{~mm}$ and the rest short-tailed with a $\mathrm{TL}=19-29 \mathrm{~mm}$ ) indicative of the presence of at least 2 species. DNA was extracted using the Qiagen DNeasy blood and tissue kit following the manufacturer's protocol. The cytochrome B (cytB) locus was amplified using primers MVZ05 (5' - CGA AGC TTG ATA TGA AAA ACC ATC GTT G) (Smith and Patton 1993) and H15915 (5' - AAC TGC AGT CAT CTC CGG TTT ACA AGA C) (Irwin et al. 1991). PCR reactions were carried out at a final volume of 20 $\mu \mathrm{L}$ containing $1 \times$ ThermoScientific DreamTaq Green Master Mix (includes $0.2 \mathrm{mM}$ dNTP and $2 \mathrm{mM} \mathrm{MgCl2}$ ), $0.5 \mu \mathrm{M}$ each primer and water and $2 \mu \mathrm{L}$ DNA template. PCR cycling conditions were $95{ }^{\circ} \mathrm{C}$ for $2 \mathrm{~min}$, followed by 35 cycles of $95{ }^{\circ} \mathrm{C} 30 \mathrm{~s}, 58^{\circ} \mathrm{C} 30 \mathrm{~s}, 72{ }^{\circ} \mathrm{C} 30 \mathrm{~s}$, and a final extension of $72^{\circ} \mathrm{C} 10 \mathrm{~min}$. Amplified products were cleaned by adding $10 \mathrm{U}$ exonuclease $\mathrm{I}$ and $1 \mathrm{U}$ shrimp alkaline phosphatase and incubating at $37^{\circ} \mathrm{C}$ for $15 \mathrm{~min}$, then $80^{\circ} \mathrm{C}$ for $15 \mathrm{~min}$. Products were Sanger sequenced on an ABI 3730XL genetic analyzer (GATC Biotech, Konstanz, Germany). Geneious v. 7.1 was used to edit the sequences. Samples were checked and cleaned for pseudogene co-amplification (identified in 1 sample with a positive cytB sequence). Sequences were compared to GenBank by carrying out a BLAST search and molecular species identification was confirmed if there was a 99-100\% match to voucher specimens of a single species in GenBank.

Large mammals ( $>2 \mathrm{~kg}$ in body weight) were surveyed annually between June and August 2006-2016, these months fall outside of the rainy season (heaviest rainfall is from October to February; Fundación Ecologista 1994). Any bias that this method introduced due to seasonal activity of mammals was logistically unavoidable. Each line transect was walked in its entirety once or twice during this period (see Appendix for details) and species tracks and signs were recorded and, where possible, identified following Reid (1997, 2009). These diurnal transect surveys began at 8 am after 48 hours of no disturbance. This non-disturbance period was to minimize bias toward nocturnal species created by an early morning start time. Although 3 local trackers were involved throughout this study, their identity varied from year to year due to availability. This was an unavoidable source of potential error due to logistical constraints and the long-term nature of the time-series. Typical walking speed was $1-2 \mathrm{~km} / \mathrm{h}$, culminating in a total of $600 \mathrm{~km}$ of transect survey in the core and $216 \mathrm{~km}$ in the buffer zone. Indirect detections were footprint tracks (a.k.a. spoor), whilst signs were any species-specific markings, for example, scat, distinctive scratch marks or food remains with identifiable tooth marks. Indirect signs also included vocalizations, particularly for arboreal species such as primates. Direct sightings of any species were also recorded but rare.

Additionally, camera traps (Bushnell Trophy Cam HD model 119477) were placed either in pairs or in triplets at locations on each transect approximately $1 \mathrm{~km}$ apart (minimum separation along transects was $200 \mathrm{~m}$ and maximum was $1.5 \mathrm{~km}$ ). Paired deployments (2014 only, were part of a pilot study) were placed $<20 \mathrm{~m}$ and ca 75 $\mathrm{m}$ perpendicular from the transect, whilst triplet deployments (2015-2016) were placed off-trail at $<20 \mathrm{~m}$, ca $150 \mathrm{~m}$ and ca $300 \mathrm{~m}$, perpendicular to the transect in an attempt to quantify differences in detection with distance from access trails. Cameras were deployed for ca 29 consecutive nights in 2014 but just 3 consecutive nights during 2015-2016 (all survey effort was approximately equal between cameras within- and between-transects within-years). This decrease in survey effort per session was to increase the number of independent sampling locations as we wanted to minimize problems associated with field of view, theft etc. Cameras were strapped to trees at approximately $30 \mathrm{~cm}$ height from the ground at locations where areas were clear from vegetation for a distance of up to $3 \mathrm{~m}$ directly in front of the camera. Camera trapping occurred at Buenos Aires during 2014 only and was not conducted at Santo Tomas, there were also occasions where cameras were stolen or malfunctioned (see Appendix for camera numbers each year). In total, 1,043 trap nights occurred in the core zone and 402 trap nights in the buffer zone. Traps were deployed upon completion of line transect surveys to minimize capturing the same individuals on cameras as detected by tracks and signs. Cameras were set to record 20 second videos with a minimum trigger interval of 1 minute. Any videos of the same species recorded within 1 hour were excluded from analyses as they may represent repeat detections.

For the camera trap survey carried out by Panthera, cameras of model Moultrie M-100 and Panthera Cams were used. Cameras were placed on human trails and mountain ridges to maximize the probability of capturing jaguar images. Between 3 and a maximum of 12 cameras were deployed at a time for 30-90 consecutive nights from 2014-2016 during both the dry and rainy season. Cameras were strapped to trees at approximately $30 \mathrm{~cm}$ height from the ground and aimed directly at trails. Only 
the species not detected by the main Operation Wallacea Ltd., camera trapping surveys have been included here for the purpose of providing a comprehensive species inventory.

Data Analysis We processed camera trap videos manually. For image organization and management, we used the camtrapR package (Niedballa et al. 2017) for R (R Core Team 2017) in RStudio (RStudio Team 2015).

We calculated indices of abundance for each survey technique separately for data collected within the core and buffer zones (for the purposes of comparison). For line transect surveys, the number of records were divided by the total kilometers surveyed in each zone and multiplied by 10 to give detections $/ 10 \mathrm{~km}$. For live trapping, we divided the number of records by the total number of trap nights in each zone multiplied by 30 nights to give detections/month for each zone. For camera trapping surveys, we divided the number of records for each zone by the total number of fractional days (to account for time of day at which cameras were deployed/collected) multiplied by 30 to give detections/month within each zone. Standardization of the units allowed direct comparison of detection rates between zones.

We calculated Shannon's Evenness Index for species community composition for small mammals using live trapping data and large mammals using tracks and signs from walked transects using the $\mathrm{R}$ package vegan (Oksanen et al. 2018). Whittaker rank abundance plots were also created using the same data. Both metrics accounted for the standardization of detection rates. We created Species Accumulation Curves for all small and large mammals pooled across survey methods to determine if our species inventory was nearing asymptote.

To assess differences in detection rates between the core and buffer zones, we conducted Wilcoxon rank sum tests on indices of abundance on data from each survey method separately. These analyses were carried out in RStudio and plotted using the package ggplot2 (Wickham 2009).

\section{Results}

Species Accumulation Curves for large mammals reached asymptote in 2014; 9 years after surveys began (Fig. 2A), not withstanding the detection of jaguar during 2016. Small mammal data have yet to reach asymptote increasing with a decelerating rate of discovery throughout the times-series with the addition of new species in virtually every year of survey.

The small mammal community (21 species) was unevenly structured being dominated by 2 species (Fig. 2B; Table 1), the Mexican deer mouse (Peromyscus mexicanus (Saussure, 1860)) with 25 records/month and Desmarest's spiny pocket mouse (Heteromys desmarestianus Gray, 1868) with 20 records/month. Of the 3 candidate Handleyomys species possibly present at the site, 2 were tentatively identified as Handleyomys cf.
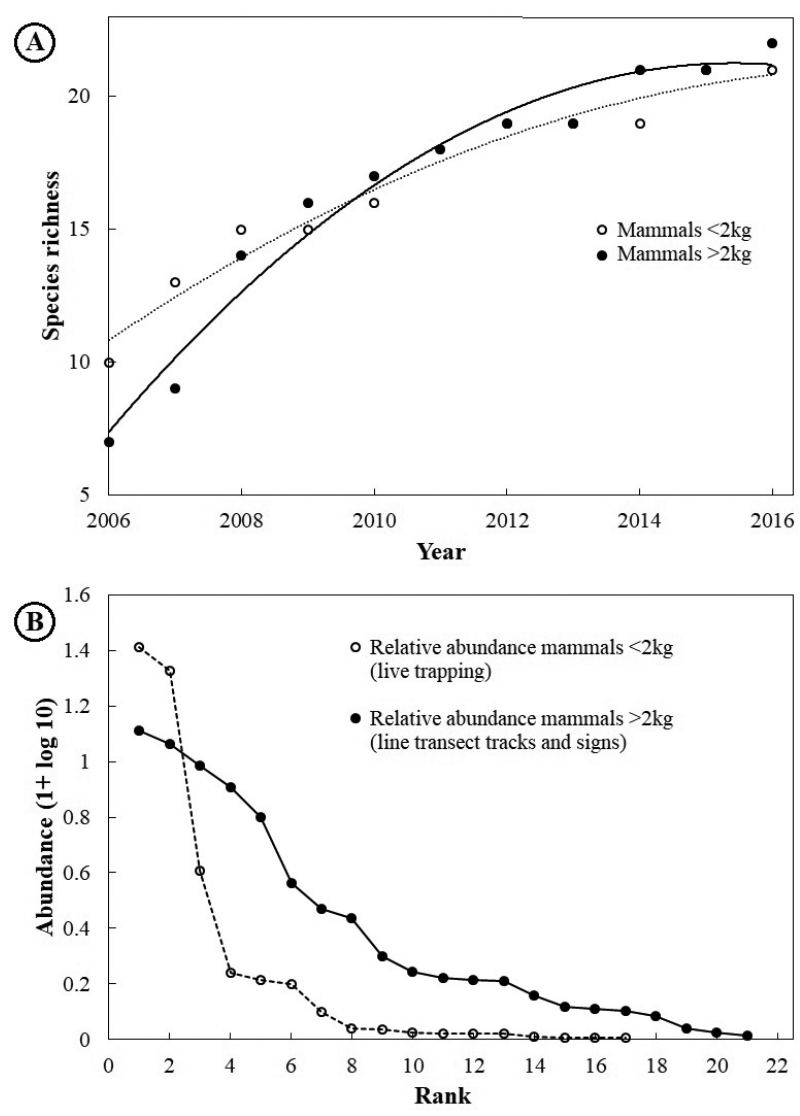

Figure 2. A. Species accumulation curves for small mammal species detections (Note: small mammals were not surveyed in 2011). B. Whittaker rank abundance plot exhibiting species richness from 2 survey methods.

rostratus and Handleyomys cf. alfaroi (Table 1); further work including molecular genetic analyses may be required to confirm these identities.

The large mammal community consisted of 22 species with a more even structure than the small mammal community, i.e. not dominated by a few common species (Fig. 2B, Table 2). The small mammal community had a Shannon index $=1.14$ compared to that of the large mammal community with a Shannon index $=2.25$ (further confirming that the small mammal community was skewed by a few hyperdominant species). Of the large mammals, the most commonly detected species included Central American red brocket deer (Mazama temama (Kerr, 1792)) with 12 records $/ 10 \mathrm{~km}$, white-nosed coati (Nasua narica (Linnaeus, 1766)) with 11 records/10 $\mathrm{km}$, Baird's tapir with 9 records $/ 10 \mathrm{~km}$ and spotted paca (Cuniculus paca (Linnaeus, 1766)) with 7 records $/ 10 \mathrm{~km}$ surveyed. Species of conservation importance included the Data Deficient Central American red brocket deer, the Near Threatened jaguar and margay (Leopardus wiedii (Schinz, 1821)) and the Endangered Baird's tapir (Fig. 3). Twelve other species recorded on camera traps are shown in Figure 3. Jaguar had previously gone unrecorded in Cusuco National Park and its detection (Table 2; Fig. 3) is strategically and geographically important within the Central American jaguar corridor.

Standardized relative indices of mammal abundance 
Table 1. Non-volant (small) mammals $<2 \mathrm{~kg}$. IUCN Red List code: DD= Data Deficient, LC = Least Concern (IUCN statuses extracted from http://www.iucnredlist.org, version 2016.3). †: there have been identifications to species level (Sciurus variegatoides and Sciurus deppei) in the study area by the author MJ, however, most were recorded just to genus only. ¥: Detection type: LT = captures/month, TS = tracks and/or signs $/ 10 \mathrm{~km}, \mathrm{DS}=$ direct sighting $/ 10 \mathrm{~km}, \mathrm{CT}=$ Camera trap detection/month, PCT = Panthera camera trap (index of abundance not included).

\begin{tabular}{|c|c|c|c|c|c|}
\hline Species recorded & Common name & $\begin{array}{l}\text { IUCN } \\
\text { status }\end{array}$ & $\begin{array}{l}\text { Core zone } \\
\text { index of } \\
\text { abundance }\end{array}$ & $\begin{array}{l}\text { Buffer zone } \\
\text { index of } \\
\text { abundance }\end{array}$ & $\begin{array}{c}\text { Detection } \\
\text { type }^{\ddagger}\end{array}$ \\
\hline \multicolumn{6}{|l|}{ RODENTIA } \\
\hline \multicolumn{6}{|l|}{ Cricetidae } \\
\hline Handleyomys cf. rostratus (Merriam, 1901) & Long-nosed rice rat & LC & 0.03 & 0.07 & LT \\
\hline Tylomys nudicaudus Peters, 1866 & Peters's climbing rat & LC & 0.04 & 0.02 & LT \\
\hline Nyctomys sumichrasti (Saussure, 1860) & Vesper rat & LC & 0.05 & 0 & LT \\
\hline Peromyscus mexicanus (Saussure, 1860) & Mexican deer mouse & LC & 13.25 & 11.51 & LT \\
\hline Handleyomys cf. alfaroi (J.A. Allen, 1891) & Alfaro's rice rat & LC & 0.49 & 0.09 & LT \\
\hline Oligoryzomys fulvescens (Saussure, 1860) & Fulvous pygmy rice rat & LC & 0.01 & 0 & LT \\
\hline Reithrodontomys cf. gracilis J.A. Allen \& Chapman, 1897 & Slender harvest mouse & LC & 0.05 & 0.21 & LT \\
\hline Scotinomys teguina (Alston, 1877) & Short-tailed singing mouse & LC & 1.25 & 1.79 & LT \\
\hline \multicolumn{6}{|l|}{ Erethizontidae } \\
\hline Sphiggurus mexicanus (Kerr, 1792) & Mexican hairy dwarf porcupine & LC & 0.02 & 0.05 & TS (DS) \\
\hline \multicolumn{6}{|l|}{ Geomyidae } \\
\hline Orthogeomys sp. & Pocket gopher & LC & 0.18 & 0.14 & TS \\
\hline \multicolumn{6}{|l|}{ Heteromyidae } \\
\hline Heteromys desmarestianus Gray, 1868 & $\begin{array}{l}\text { Desmarest's spiny pocket } \\
\text { mouse }\end{array}$ & LC & 14.53 & 5.61 & LT \\
\hline \multicolumn{6}{|l|}{ Muridae } \\
\hline Mus musculus Linnaeus, 1758 & House mouse & LC & 0 & 0.05 & LT \\
\hline \multicolumn{6}{|l|}{ Sciuridae $^{\dagger}$} \\
\hline Sciurus sp. & Squirrel & LC & 2.39 & 1.57 & CT \\
\hline \multicolumn{6}{|l|}{ DIDELPHIMORPHIA } \\
\hline \multicolumn{6}{|l|}{ Didelphidae } \\
\hline Didelphis marsupialis Linnaeus, 1758 & Black-eared opossum & LC & 0.27 & 0.47 & LT \\
\hline Marmosa mexicana Merriam, 1897 & Mexican mouse opossum & LC & 0.28 & 0.35 & LT \\
\hline \multicolumn{6}{|l|}{ LAGOMORPHA } \\
\hline \multicolumn{6}{|l|}{ Leporidae } \\
\hline Sylvilagus gabbi gabbi (J.A. Allen, 1877) & Tapeti rabbit & LC & 0.01 & 0 & LT \\
\hline \multicolumn{6}{|l|}{ CARNIVORA } \\
\hline \multicolumn{6}{|l|}{ Mustelidae } \\
\hline \multirow[t]{2}{*}{ Mustela frenata Lichtenstein, 1831} & Long-tailed weasel & LC & 0.05 & 0 & LT \\
\hline & & & 0.03 & 0.15 & $\mathrm{CT}$ \\
\hline \multicolumn{6}{|l|}{ Procyonidae } \\
\hline Bassariscus sumichrasti (Saussure, 1860) & Cacomistle & LC & NA & NA & PCT \\
\hline \multicolumn{6}{|l|}{ EULIPOTYPHLA } \\
\hline \multicolumn{6}{|l|}{ Soricidae } \\
\hline Cryptotis mccarthyi Woodman, 2015 & Omoa broad-clawed shrew & DD & NA & NA & LT \\
\hline Cryptotis merriami Choate, 1970 & Merriam's small-eared shrew & LC & NA & NA & LT \\
\hline Cryptotis orophila (J.A. Allen, 1895) & Central American least shrew & DD & NA & NA & LT \\
\hline
\end{tabular}

did not differ significantly between the core than buffer zones (due to the high prevalence of zero detections and wide variation in detection at sites within each zone) but were consistently higher within the core and buffer zone, specifically, mammal tracks and signs on line transect surveys were $30 \%$ higher $(\mathrm{W}=219.5, \mathrm{P}=0.79)$, camera trap detections were $46 \%$ higher $(\mathrm{W}=127, \mathrm{P}=0.56)$ and small mammal live trapping capture rates were $33 \%$ higher $(\mathrm{W}=128, \mathrm{P}=0.58)$ within the core compared to buffer zone (Fig. 4).

\section{Identification}

Species accounts of uncommon species ( $<10$ records) or those where identification required some further explana- tion are included below for reference with the exception of the house mouse (Mus musculus) as it is sufficiently recognized globally.

\section{Oligoryzomys fulvescens (Saussure, 1860)}

Material examined. Honduras, Cortés, Cusuco National Park, 15³1'24" N, 088 17'19" W, Neil Reid, 2012, Table 1.

Longer tail than other Central American mice of similar size. The differences in ratio of toe lengths as well as differences in upper incisors with similar harvest mouse species (Reid 2009) were used to identify this species.

\section{Sphiggurus mexicanus (Kerr, 1792)}


Table 2. Non-volant (medium-sized to large) mammals $>2 \mathrm{~kg}$. Station codes: $\mathrm{BA}=$ Buenos Aires, $\mathrm{BC}=$ base camp, $\mathrm{CA}=\mathrm{Cantiles}, \mathrm{CO}=\mathrm{Cor}-$ tecito, $\mathrm{CP}=$ Capuca, DA = Danto, GU = Guanales, ST = Santo Tomas. IUCN Red List codes: DD = Data deficient, LC = Least Concern, NT = Near Threatened and EN = Endangered (IUCN statuses extracted from http://www.iucnredlist.org, version 2016.3). t: Index of abundance not stated for those records provided by Panthera. ¥: Detection type: $\mathrm{LT}=$ captures $/ \mathrm{month}, \mathrm{TS}=$ tracks and signs $/ 10 \mathrm{~km}, \mathrm{DS}=$ direct sighting $/ 10$ $\mathrm{km}, \mathrm{CT}=$ Camera trap detections/month, PCT = Panthera camera trap.

\begin{tabular}{|c|c|c|c|c|c|}
\hline Species recorded & Common name & $\begin{array}{l}\text { IUCN } \\
\text { sta- } \\
\text { tus }\end{array}$ & $\begin{array}{l}\text { Core zone } \\
\text { index of } \\
\text { abundance }^{\dagger}\end{array}$ & $\begin{array}{l}\text { Buffer zone } \\
\text { index of } \\
\text { abundance }\end{array}$ & $\begin{array}{c}\text { Type of } \\
\text { detection }\end{array}$ \\
\hline \multicolumn{6}{|l|}{ PRIMATES } \\
\hline \multicolumn{6}{|l|}{ Atelidae } \\
\hline Alouatta palliata (Gray, 1849) & Mantled howler monkey & LC & 1.5 & 0.46 & TS (DS) \\
\hline \multicolumn{6}{|l|}{ CARNIVORA } \\
\hline \multicolumn{6}{|l|}{ Canidae } \\
\hline Urocyon cinereoargenteus (Schreber, 1775) & Gray fox & LC & 0.13 & 0.09 & TS \\
\hline \multicolumn{6}{|l|}{ Felidae } \\
\hline Leopardus wiedii (Schinz, 1821) & Margay & NT & $\begin{array}{l}0.04 \\
0.26\end{array}$ & $\begin{array}{c}0 \\
0.22\end{array}$ & $\begin{array}{l}\text { TS } \\
C T\end{array}$ \\
\hline Leopardus pardalis (Linnaeus, 1758) & Ocelot & LC & 0.39 & 0.28 & TS \\
\hline Herpailurus yagouaroundi (É. Geoffroy-Hilaire, 1803) & Jaguarundi & LC & $\begin{array}{l}0.13 \\
0.03\end{array}$ & $\begin{array}{c}0.14 \\
0\end{array}$ & $\begin{array}{l}\text { TS } \\
\text { CT }\end{array}$ \\
\hline Panthera onca Linnaeus, 1758 & Jaguar & NT & NA & & PCT \\
\hline \multicolumn{6}{|l|}{ Mephitidae } \\
\hline Conepatus semistriatus (Boddaert, 1785) & Striped hog-nosed skunk & LC & 0.25 & 0.74 & TS (DS) \\
\hline \multicolumn{6}{|l|}{ Mustelidae } \\
\hline Eira barbara (Linnaeus, 1758) & Tayra & LC & $\begin{array}{l}0.11 \\
0.03\end{array}$ & $\begin{array}{l}0.19 \\
0.08\end{array}$ & $\begin{array}{l}\text { TS } \\
\text { CT }\end{array}$ \\
\hline Galictis vittata (Schreber, 1776) & Greater grison & LC & NA & NA & PCT \\
\hline \multicolumn{6}{|l|}{ Procyonidae } \\
\hline Potos flavus (Schreber, 1774) & Kinkajou & LC & $\begin{array}{l}0.39 \\
0.06\end{array}$ & $\begin{array}{l}0.23 \\
0.15\end{array}$ & $\begin{array}{l}\mathrm{TS} \\
\mathrm{CT}\end{array}$ \\
\hline Nasua narica (Linnaeus, 1766) & White-nosed coati & LC & $\begin{array}{l}6.09 \\
0.72\end{array}$ & $\begin{array}{l}4.44 \\
0.08\end{array}$ & $\begin{array}{l}\text { TS } \\
\text { CT }\end{array}$ \\
\hline Procyon lotor (Linnaeus, 1758) & Northern raccoon & LC & 0.30 & 0.14 & TS \\
\hline \multicolumn{6}{|l|}{ CETARTIODACTYLA } \\
\hline \multicolumn{6}{|l|}{ Cervidae } \\
\hline Mazama temama (Kerr, 1792) & Central American red brocket & DD & $\begin{array}{l}6.36 \\
0.63\end{array}$ & $\begin{array}{l}5.56 \\
0.60\end{array}$ & $\begin{array}{l}\mathrm{TS} \\
\mathrm{CT}\end{array}$ \\
\hline Odocoileus virginianus (Zimmermann, 1780) & White-tailed deer & LC & $\begin{array}{c}0.86 \\
0\end{array}$ & $\begin{array}{l}0.88 \\
0.15\end{array}$ & $\begin{array}{l}\text { TS } \\
\text { CT }\end{array}$ \\
\hline \multicolumn{6}{|l|}{ Tayassuidae } \\
\hline Pecari tajacu (Linnaeus, 1758) & Collared peccary & LC & $\begin{array}{l}0.43 \\
0.58\end{array}$ & $\begin{array}{c}0.32 \\
0\end{array}$ & $\begin{array}{l}\text { TS } \\
\text { CT }\end{array}$ \\
\hline \multicolumn{6}{|l|}{ RODENTIA } \\
\hline \multicolumn{6}{|l|}{ Cuniculidae } \\
\hline Cuniculus paca (Linnaeus, 1766) & Spotted paca & LC & $\begin{array}{l}3.97 \\
1.73\end{array}$ & $\begin{array}{l}3.10 \\
0.22\end{array}$ & $\begin{array}{l}\text { TS } \\
\text { CT }\end{array}$ \\
\hline \multicolumn{6}{|l|}{ Dasyproctida } \\
\hline Dasyprocta punctata (Gray, 1842) & Agouti & LC & $\begin{array}{l}0.45 \\
0.06\end{array}$ & $\begin{array}{l}0.19 \\
0.08\end{array}$ & $\begin{array}{l}\mathrm{TS} \\
\mathrm{CT}\end{array}$ \\
\hline \multicolumn{6}{|l|}{ CINGULATA } \\
\hline \multicolumn{6}{|l|}{ Chlamyphoridae } \\
\hline Cabassous centralis (Miller, 1899) & $\begin{array}{l}\text { Northern naked-tailed } \\
\text { Armadillo }\end{array}$ & DD & NA & NA & PCT \\
\hline \multicolumn{6}{|l|}{ Dasypodidae } \\
\hline Dasypus novemcinctus Linnaeus, 1758 & Nine-banded armadillo & LC & $\begin{array}{l}3.22 \\
0.09\end{array}$ & $\begin{array}{l}2.08 \\
0.08\end{array}$ & $\begin{array}{l}\text { TS } \\
\text { CT }\end{array}$ \\
\hline \multicolumn{6}{|l|}{ DIDELPHIMORPHIA } \\
\hline Didelphis virginiana (Kerr, 1792) & Virginia opossum & LC & $\begin{array}{l}0.03 \\
1.54 \\
0.43 \\
\end{array}$ & $\begin{array}{l}0.05 \\
1.11 \\
0.60 \\
\end{array}$ & $\begin{array}{l}\text { LT } \\
\text { TS } \\
\text { CT }\end{array}$ \\
\hline \multicolumn{6}{|l|}{ PILOSA } \\
\hline \multicolumn{6}{|l|}{ Myrmecophagidae } \\
\hline Tamandua mexicana (Saussure, 1860) & Northern tamandua & LC & 0.01 & 0 & LT \\
\hline $\begin{array}{l}\text { PERISSODACTYLA } \\
\text { Tapiridae }\end{array}$ & & & & & \\
\hline Tapirus bairdii (Gill, 1865) & Baird's tapir & EN & $\begin{array}{l}7.22 \\
0.17\end{array}$ & $\begin{array}{c}1.44 \\
0\end{array}$ & $\begin{array}{l}\text { TS } \\
\text { CT }\end{array}$ \\
\hline
\end{tabular}




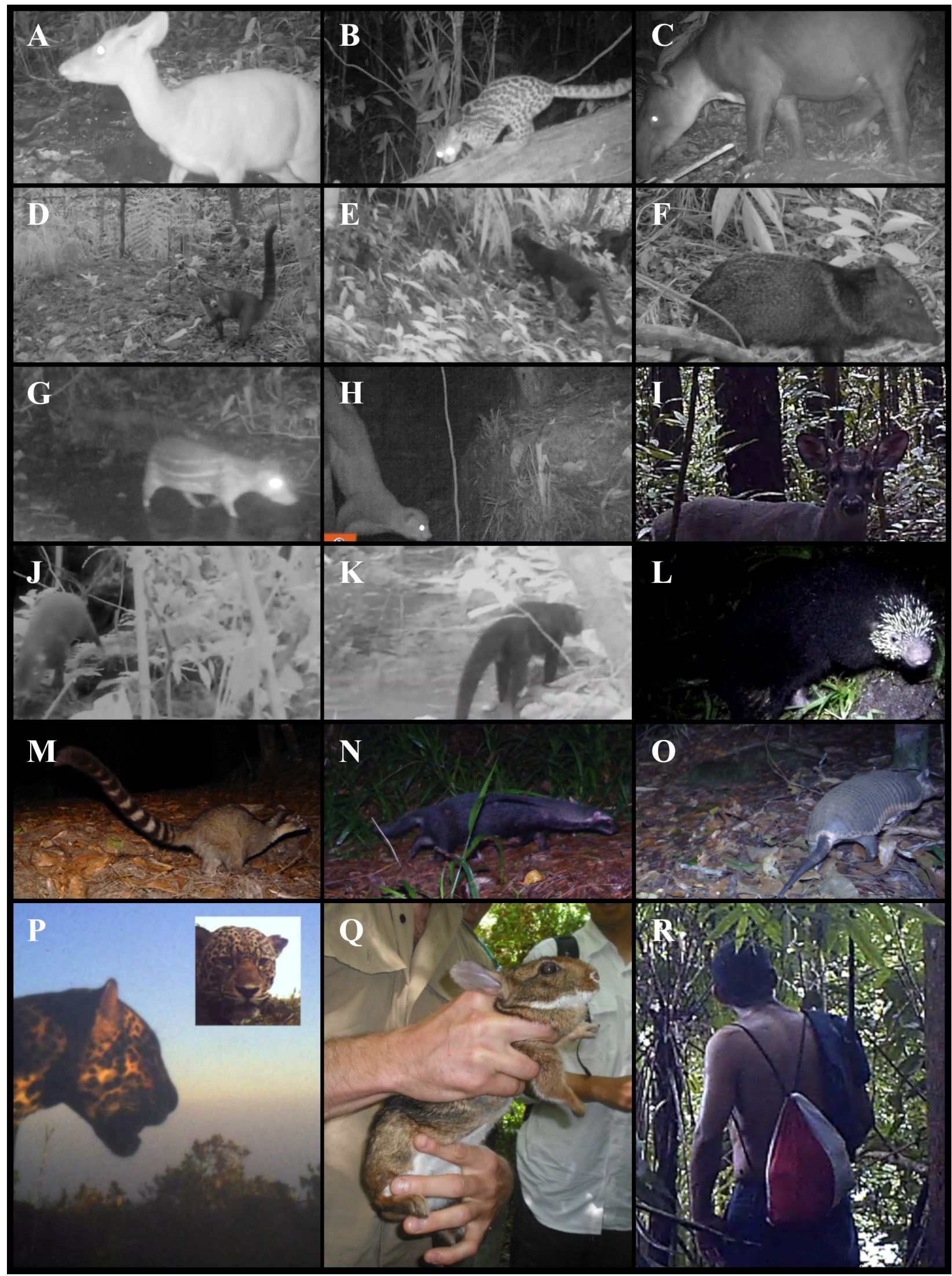

Figure 3. Photographs of key species detections including: (A) Data Deficient Central American red brocket deer (Mazama temama), (B) Near Threatened margay (Leopardus wiedii), and (C) Endangered Baird's tapir (Tapirus bairdii). Further photographs of species detected on camera traps including: (D) White-nosed coati (Nasua narica), (E) Jaguarundi (Herpailurus yagouaroundi), (F) Collared peccary (Pecari tajacu), (G) Paca (Cuniculus paca), (H) Kinkajou (Potos flavus), (I) White-tailed deer (Odocoileus virginianus), (J) Agouti (Dasyprocta punctata), (K) Tayra (Eira barbara), (L) Mexican hairy dwarf porcupine (Sphiggurus mexicanus) [photo credit: Katie Rapson], (M) Cacomistle (Bassariscus sumichrasti) [Photo credit: Panthera Honduras], (N) Greater grison (Galictis vittata) [photo credit: Panthera Honduras], (0) Northern naked-tailed armadillo (Cabassous centralis) [photo credit: Panthera Honduras], (P) Jaguar (Panthera onca) caught by camera trap at 2,200 m a.s.l. [photo credit: Panthera Honduras], (Q) Tapeti rabbit (Sylvilagus gabbi gabbi) when removed from a trap in 2010 [photo credit: Rachael Forster], (R) hunter carrying weapon stands in front of camera trap. 

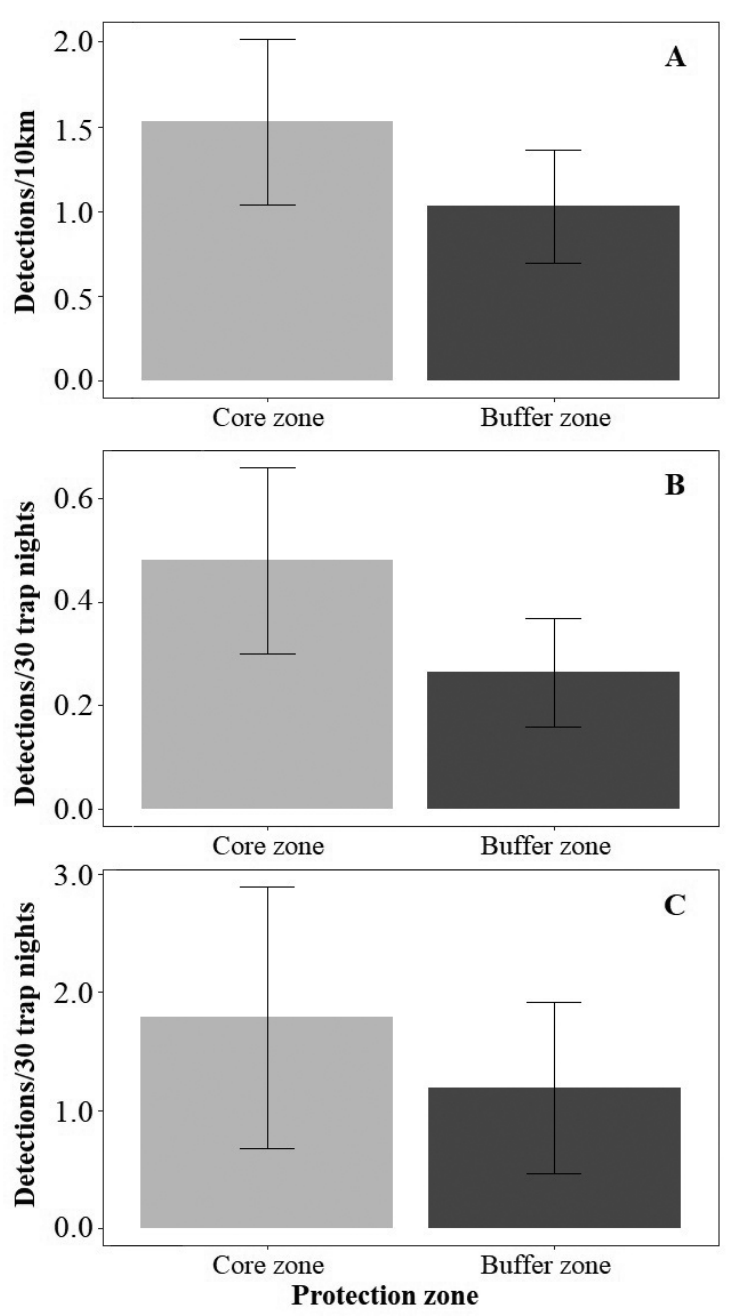

Figure 4. A. Relative abundance of tracks and signs on line transects surveys $/ 10 \mathrm{~km}$ in the core vs buffer. B. Relative camera trap detections/month. C. Relative capture rate by live trapping/month. All 3 plots include standard error bars.

Material examined. Honduras, Cortés, Cusuco National

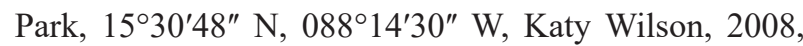
Table 2, Figure 3L.

The only species of porcupine found in Honduras (Reid 2009).

\section{Bassariscus sumichrasti (Saussure, 1860)}

Material examined. Honduras, Cortés, Cusuco National Park, Franklin Castañeda, 2014, Table 2; Figure 3M.

From camera trap footage it was distinguished from other similar species due to its bushier and very clearly banded tail (Reid 2009).

\section{Tylomys nudicaudus Peters, 1866}

Material examined. Honduras, Cortés, Cusuco National Park, 15³1'24" N, 088¹7'19" W, David Beaune, 2008, Table 1.

Distinguished from other species of its genus by its geographic range (Reid 2009), it differed from other similar species due to a white-tipped tail (Reid 2009).

\section{Nyctomys sumichrasti (Saussure, 1860)}

Material examined. Honduras, Cortés, Cusuco National

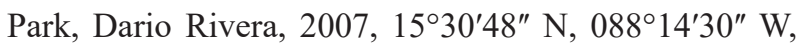
Table 1.

Geographically distant to similar species (Reid 2009).

Reithrodontomys gracilis J.A. Allen \& Chapman, 1897

Material examined. Honduras, Cortés, Cusuco National Park, 15³1'24" N, 088 17'19" W, Dario Rivera, 2007, Table 1.

Small size distinguished it from other very similar species (Reid 2009).

\section{Mustela frenata (Lichtenstein, 1831)}

Material examined. Honduras, Cortés, Cusuco National Park, $15^{\circ} 29^{\prime} 19^{\prime \prime}$ N, $088^{\circ} 14^{\prime} 03^{\prime \prime}$ W, Dario Rivera, 2007, Table 1.

Elongated body and narrow tail, easily distinguishable from similar species due to much smaller size and clearly different tail to squirrel species (Reid 2009; screen-grabs of camera trap recordings from 2015 or 2016 have not been included as they are not clear as still images).

\section{Tamandua mexicana (Saussure, 1860)}

Material examined. Honduras, Cortés, Cusuco National Park, $15^{\circ} 30^{\prime} 48^{\prime \prime} \mathrm{N}, 088^{\circ} 14^{\prime} 30^{\prime \prime}$ W, Dario Rivera, 2007, Table 2.

Nothing else in the region with which it may have been mistaken (Reid 2009).

\section{Galictis vittata (Schreber, 1776)}

Material examined. Honduras, Cortés, Cusuco National Park, Franklin Castañeda, 2014, Table 2; Figure 3N.

The gray color distinguishes this species easily from other, similar species (Reid 2009).

\section{Cabassous centralis (Miller, 1899)}

Material examined. Honduras, Cortés, Cusuco National Park, Franklin Castañeda, 2014, Table 2; Figure 3 O.

Flatter body shape than that of Dasypus novemcinctus, with a shorter, less robust tail (Reid 2009).

\section{Panthera onca (Linnaeus, 1758)}

Material examined. Honduras, Cortés, Cusuco National Park, Franklin Castañeda, 2016, Table 2; Figure 3P.

Shape and markings are unmistakable even with other spotted cat species found in the area (Reid 2009).

\section{Sylvilagus gabbi gabbi (J.A. Allen, 1877)}

Material examined. Honduras, Cortés, Cusuco National Park, 15²9'47" N, 088 $12^{\prime} 43^{\prime \prime}$ W, Rachael Forster, 2010, Table 1, Figure 3Q.

The only lagomorph whose range falls across Cusuco 
National Park. Figure 3Q shows color patterns which were considered along with size (Reid 2009). Originally described as Sylvilagus brasiliensis (Linnaeus, 1758), but taxonomic work has led to its reclassification as $S$. gabbi with those in the study area being subspecies gabbi (Allen 1877, Ruedas and Salazar-Bravo 2007).

\section{Handleyomys alfaroi (J.A. Allen 1891)}

Material examined. Honduras, Cortés, Cusuco National Park, 15²9'47" N, 088 $12^{\prime} 43^{\prime \prime}$ W, Rachael Forster, 2010, Table 1.

The genus Handleyomys, notwithstanding undescribed cryptic species, currently contains 8 described species: $H$. chapmani, $H$. fuscatus, $H$. intectus, $H$. melanotis and $H$. rhabdops, but only $3-H$. alfaroi, $H$. rostratus and $H$. saturatior - have ranges that include any part of Honduras and only 2 ( $H$. alfaroi and $H$. rostratus) have ranges which included Cusuco National Park (both IUCN range data and Wilson, Lacher and Mittermeier 2017).

Handleyomys alfaroi and $H$. saturatior could conceivably be confused due to their similar body size (HB 90-106 $\mathrm{mm}$ and 90-103 $\mathrm{mm}$ respectively) but their tails are fairly diagnostic with the former having a shorter tail (89-101 mm) than the latter (105-122 mm). Additionally, the former has a lighter coat color (dull yellow-brown) compared to the latter (dark brown very heavily mixed with black).

\section{Handleyomys rostratus (Merriam, 1901)}

Material examined. Honduras, Cortés, Cusuco National

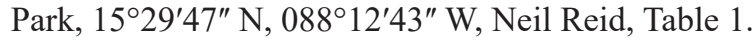

Following the above, this species is notably larger than the other 2 species of the genus found in Honduras (HB 100-140mm) and distinctly colored, being lighter reddish brown with a white venter and well-defined countershading absent from the other 2 candidate species.

\section{Cryptotis merriami Choate, 1970}

Material examined. Honduras, Cortés, Cusuco National Park, $1^{\circ} 29^{\prime} 47.1^{\prime \prime}$ N, $088^{\circ} 12^{\prime} 42.7^{\prime \prime}$ W, Neil Reid, 2012, NMS.Z.2018.80.6, Tables 1, 3, Figure 5.

\section{Cryptotis orophilus (J.A. Allen, 1895)}

Material examined. Honduras, Cortés, Cusuco National

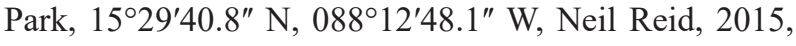
NMS.Z.2018.80.3, Tables 1, 3; Figure 5.

\section{Cryptotis mccarthyi Woodman, 2015}

Material examined. Honduras, Cortés, Cusuco National Park, 15³1'11.5" N, 088 $13^{\circ} 55.0^{\prime \prime}$ W, Neil Reid, 2015, NMS.Z.2018.80.4, Tables 1, 3, Figure 5.

Seven out of the 10 Cryptotis specimens taken by lethal sampling had successfully amplified DNA and were sequenced for the gene CytB. For all sequences, a greater than $99 \%$ species match could be made to voucher specimens sequenced by Baird et al. (2018) from specimens collected in Honduras (Table 3). Of those described as short-tailed, 2 species were identified: C. merriami and C. orophilus, whilst those described as long-tailed were identified as C. mccarthyi (Table 1, Fig. 5).

Four species were detected by indirect methods only. Local trackers identified ocelot (Leopardus pardalis Linnaeus 1758) using tracks and feces, Northern raccoon (Procyon lotor (Linnaeus, 1758)) and Gray fox (Urocyon cinereoargenteus (Schreber, 1775)) using tracks and evidence of food and gopher (Orthogeomys sp.) by means of burrows and digging spoil.

Sixteen species whose IUCN range polygons included Cusuco National Park went undetected (Table 4). Notable non-detections included Geoffroy's spider monkey (Ateles geoffroyi Kuhl, 1820), puma (Puma concolor (Linnaeus, 1771)) and giant anteater (Myrmecophaga tridactyla Linnaeus, 1758). The park falls within the IUCN range polygon for the Neotropical river otter (Lontra longicaudis (Olfers, 1818)), but this species was excluded from Table 4 due to the unsuitability of high altitude habitats i.e. all streams and rivers within the park are small $(<3 \mathrm{~m}$ wide) and fishless (confirmed by electrofishing) as they are above impassable waterfalls.

\section{Discussion}

Cusuco National Park has previously been considered an important Protected Area (Le Saout et al. 2013), most notably for Baird's tapir, but also strategically positioned within the Central American jaguar corridor despite no previous species records from the region (Petracca et al. 2017, Wultsch et al. 2016, Schank et al. 2017). This study

Table 3. Shrew species identification matches for seven Cryptotis specimens using CytB sequencing. Specimens are available at the Royal Museum of Scotland, Edinburgh.

\begin{tabular}{llccc}
\hline Specimen no. & Species match on GenBank & \% pairwise identity & $\begin{array}{c}\text { Length of pairwise match } \\
\text { (bp) }\end{array}$ & $\begin{array}{c}\text { GenBank accession number } \\
\text { of best match }\end{array}$ \\
\hline 08 & C. merriami & $99.7 \%$ & 906 & MF158110 \\
12 & C. merriami & $99.9 \%$ & 1070 & MF158112 \\
13 & C. orophilus & $99.5 \%$ & 497 & MF158113 \\
$18^{*}$ & C. maccarthyi & $100.0 \%$ & 1117 & MF158096 \\
22 & C. merriami & $99.8 \%$ & 911 & MF158108 \\
23 & C. merriami & $100.0 \%$ & 837 & MF158110 \\
24 & C. merriami & $99.4 \%$ & \\
\hline
\end{tabular}

* Mixed sequence - sequence match carried out after excluding the pseudogene. 


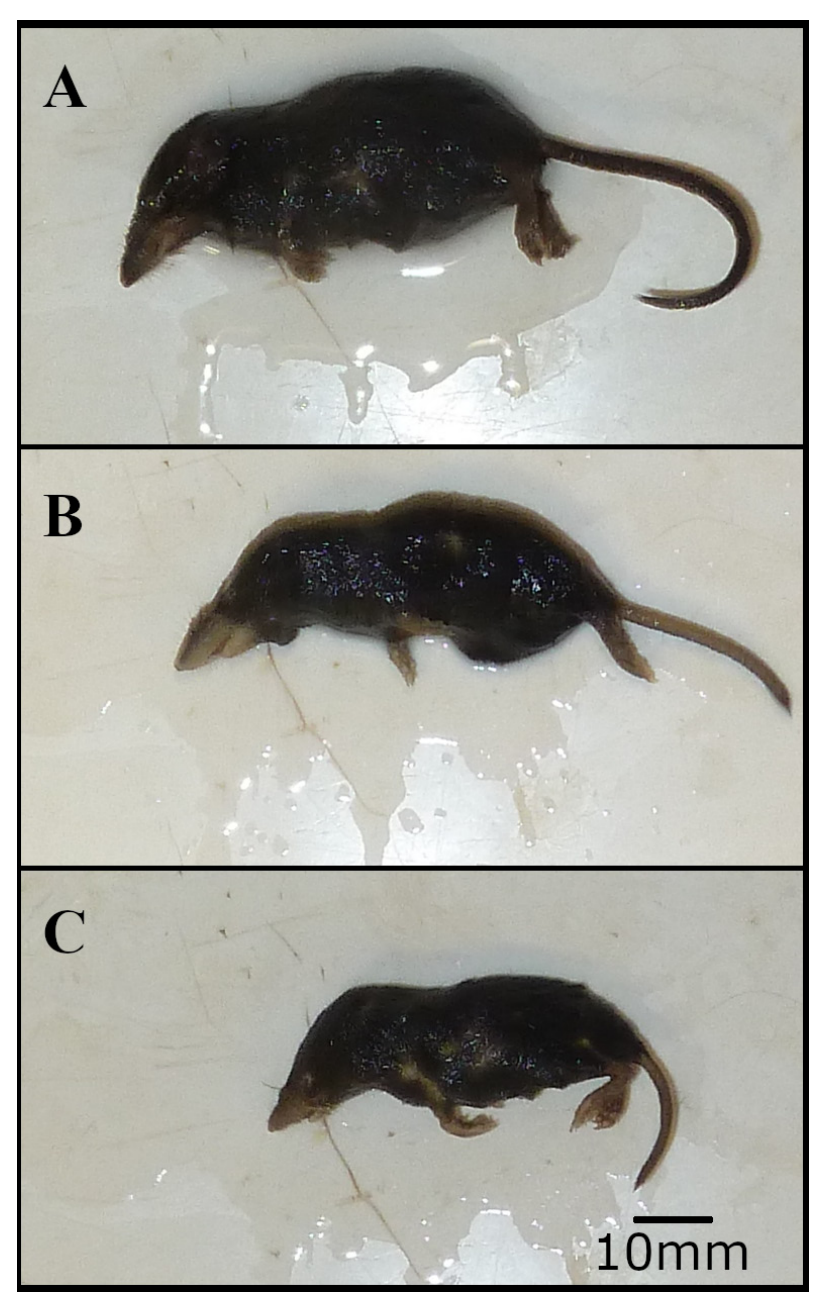

Figure 5. Three example specimens of (A) Cryptotis mccarthyi (TL $=50 \mathrm{~mm}),(\mathbf{B})$ C. merriami $(\mathrm{TL}=27-29 \mathrm{~mm})$ and $(\mathbf{C})$ C. orophilus $(\mathrm{TL}=21 \mathrm{~mm})$ identified using molecular genetic analysis of CytB sequences.

represents the first comprehensive species inventory for the park and we confirm the presence of jaguar for the first time. Cusuco National Park lies close to the city of San Pedro Sula (Fig. 1) and is highly vulnerable to human disturbance, highlighting the importance of conservation action in the region to protect its diverse mammal fauna.

A camera trapping species inventory from Río Plátano Biosphere Reserve, Honduras (Gonthier and Castañeda 2013) suggests similar species richness to Cusuco National Park. Detection of $79 \%$ of expected meso-to-large mammals within Cusuco National Park is comparable to other surveys of similar sized species in the Neotropics (Tobler et al. 2008, Cove et al. 2013). Moreover, the asymptote for our large mammal list suggests a near complete species list whilst increases in small mammals may suggest that further species remain to be described; this is most notable for cryptic species (as in the Cryptotis shrews identified here) which require molecular genetic methods.

Indices of relative abundance accounted for survey effort based upon either distance (i.e. for line transects) or duration (i.e. for camera and live trapping). Nevertheless, species-specific detections may have been subject to sources of methodological error or bias as some species may be more easily detected using certain methods, for example, Baird's tapir may leave abundant, long-lasting tracks and signs due to their weight compared to smaller, lighter-footed common species such as paca.

Cusuco National Park has zonal protection with mammal abundances greater within the core zone, which has greater protected status, than within the buffer zonevulnerable to some level of disturbance and within which there are a number of villages including Buenos Aires and Santo Tomas. Disturbance of intact forest includes fuel wood collection and hunting by people. Hunting platforms (horizontal beams lashed between 2 trees approximately $3 \mathrm{~m}$ above ground level on which hunters can perch at dawn and dusk) are regularly encountered throughout the park including within the core zone (pers. obs.), suggesting that some level of human impact may have already occurred within the most pristine areas. Hunters carrying guns were also observed on camera traps (Fig. 3R), whilst distant gunshots have been heard during surveys (pers. obs.). This is in spite of the fact that ongoing deforestation and hunting within Cusuco National Park has resulted in the deployment of army patrols and latterly, park wardens. Nevertheless, zonal differences in mammal abundance suggests that maintaining and enforcing the park's protected status is important. Other studies suggest that where protected areas are smaller than the core zone of Cusuco National Park, mammal populations cannot be sustained and are vulnerable to local extirpation (Ortiz-Lozada et al. 2017).

Large mammal community composition of Cusuco National Park was comparable (taking into account species' known ranges) to those found in other Central American reserves (Thornton et al. 2011, Cove et al 2013, Tobler et al. 2008, Gonthier and Castañeda 2013) with the notable exception of Geoffroy's spider monkey and puma (Thornton et al. 2011, Tobler et al. 2008, Gonthier and Castañeda 2013), which were absent. Historical anecdotal evidence from interviews with local people (in the villages of Buenos Aires and Bañaderos) suggest Geoffroy's spider monkey was once present and indeed numerous but has not been seen for some time. The lack of records in Cusuco National Park suggests the species is now locally extirpated. Howler monkeys, by comparison, remain widespread in the park as they have a more adaptable diet and are less disturbed by forest fragmentation (Lenz et al. 2014) whilst becoming habituated to nearby human activity (McKinney et al. 2015). Moreover, their meat is reputed to have a bad flavor and they are not the focal target of hunting (pers. obs.). Anecdotal reports of puma remain unverified and they are known to avoid areas with human settlements (Foster et al. 2010).

It is noteworthy, that whilst ocelot tracks and signs have been detected within Cusuco National Park, no direct detections have been made via camera trapping. It is odd given the species' generally high abundance throughout Central and South America (Dillon and Kelly 2008, Davis et al. 2011), especially given that jaguar, a 
Table 4. Non-volant mammalian species not detected in Cusuco National Park despite its inclusion within their IUCN range polygon. IUCN Red List codes: DD = Data deficient, LC = Least Concern, VU = Vulnerable and EN = Endangered (IUCN statuses extracted from http://www. iucnredlist.org, version 2016.3).

\begin{tabular}{|c|c|c|}
\hline Species & Common name & IUCN status \\
\hline \multicolumn{3}{|l|}{ PRIMATES } \\
\hline \multicolumn{3}{|l|}{ Atelidae } \\
\hline Ateles geoffroyi (Kuhl, 1820) & Geoffroy's spider monkey & EN \\
\hline \multicolumn{3}{|l|}{ CARNIVORA } \\
\hline \multicolumn{3}{|l|}{ Canidae } \\
\hline Canis latrans (Say, 1823) & Coyote & LC \\
\hline \multicolumn{3}{|l|}{ Felidae } \\
\hline Puma concolor (Linnaeus, 1771) & Puma & LC \\
\hline \multicolumn{3}{|l|}{ Mephitidae } \\
\hline Spilogale angustifrons (Howell, 1902) & Southern spotted skunk & LC \\
\hline \multicolumn{3}{|l|}{ RODENTIA } \\
\hline \multicolumn{3}{|l|}{ Cricetidae } \\
\hline Oryzomys couesi (Alston, 1877) & Coues' rice rat & LC \\
\hline Ototylomys phyllotis (Merriam, 1901) & Big-eared climbing rat & LC \\
\hline Sigmodon hirsutus (Burmeister, 1854) & Southern cotton rat & LC \\
\hline \multicolumn{3}{|l|}{ Heteromyidae } \\
\hline Heteromys salvini (Thomas, 1893) & Salvin's spiny pocket mouse & LC \\
\hline \multicolumn{3}{|l|}{ PILOSA } \\
\hline \multicolumn{3}{|l|}{ Cyclopedidae } \\
\hline Cyclopes didactylus (Linnaeus, 1758) & Silky anteater & LC \\
\hline \multicolumn{3}{|l|}{ Myrmecophagidae } \\
\hline Myrmecophaga tridactyla (Linnaeus, 1758) & Giant anteater & VU \\
\hline \multicolumn{3}{|l|}{ DIDELPHIMORPHIA } \\
\hline \multicolumn{3}{|l|}{ Didelphidae } \\
\hline Caluromys derbianus (Waterhouse, 1841) & Derby's woolly opossum & LC \\
\hline Chironectes minimus (Zimmermann, 1780) & Water opossum & LC \\
\hline Marmosa alstoni (J.A. Allen, 1900) & Alston's mouse opossum & LC \\
\hline Philander opossum (Linnaeus 1758) & Gray four-eyed opossum & LC \\
\hline
\end{tabular}

generally rare big cat has been recorded on camera. Ocelot diet includes small mammals, birds and reptiles, but also includes larger sized prey ( $>800 \mathrm{~g}$ ) species, such as agoutis, armadillos and pacas (Sunquist and Sunquist 2002, Moreno et al. 2006, Bianchi et al. 2010); the latter of which is notably common in Cusuco National Park. It has also been shown to be present elsewhere in Honduras (Gonthier and Castañeda 2013).

Relative to other areas in Central America, we record notably low detection rates of agouti (Cove et al. 2013) which may be a consequence of hunting for bushmeat (Roger Alvarengo pers. comm.). However, unexpected low detections of the genus Dasyprocta by camera trapping has been shown elsewhere in South America despite high detection in transect surveys (Munari et al. 2011). However, the lack of agouti in Cusuco National Park as a prey base for ocelot may well help to explain the predator's absence as well.

Other mammal species may have gone unrecorded due to our sampling methods. For example, arboreal deployment of live traps would be required to record tree-living small mammals such as climbing rats (e.g. the big-eared climbing rat Ototylomys phyllotis Merriam 1901) or canopy dwelling species (for example, to confirm the presence of Geoffroy's spider monkey). Some species occupying habitats which are difficult to survey e.g. fossorial species like gophers or aquatic species that rarely leave the water's edge such as water mice (Rheomys sp.) may require focal sampling techniques.

A population viability model for Baird's tapir within Cusuco suggested that recent declines may lead to local extirpation (McCann et al. 2012). No evidence of tapir presence was detected in 2016 for the first time during annual surveys over the last decade, supporting the assertion that its population may indeed be reaching the lower threshold for detection. Moreover, the only evidence for the presence of the species detected since the completion of this survey, was a skeleton found during 2017 which showed signs that it had been hunted (pers. obs.).

During June 2016, a single jaguar was recorded for the first time within Cusuco National Park (Fig. 3P) in the "bosque enano" (dwarf forest) at 2,200 m above sea level (see https://www.panthera.org/blog/2016/06/15/surprise2200-meters). Jaguars are typically associated with areas providing access to large-bodied prey; though in some regions such as Belize they may take smaller prey such as armadillos (Foster et al. 2010). In Cusuco National Park, prey likely includes tapir, red brocket, white-tailed deer, peccaries and conceivably domestic stock such as cattle in the north-west of the park, where deforestation 
has given way to pockets of agriculture including pasture. Conservation, both political lobbying and on-theground management, can only be effective with full knowledge of what species are present, their distribution and relative abundance. Here, we report a comprehensive species list of non-volant terrestrial mammals for Cusuco National Park including the presence of jaguar. Data on the impact of designation, disturbance and frequency of mammal detection may help to direct future conservation policy. We advise continued annual monitoring activity to provide a robust time-series, allowing temporal trends in occupancy and abundance to be investigated. Particular focus should be on those species vulnerable to exploitation for bushmeat by hunting as well as bioindicators such as top predators including jaguar. We also advise that consideration should be given to conducting an IUCN Regional Red Listing exercise (see http:// www.iucnredlist.org/about/publication/regional-redlists\#Americas) for the mammals of Honduras.

\section{Acknowledgements}

We are grateful to all Operation Wallacea Ltd staff and students. We thank Mito, Roger and Lucas whose local knowledge and species identification skills were invaluable. Annual contributions to the sightings database were contributed to by (listed in alphabetical order of surname): Alexandra Andino, Mandy Apps, Sara Aubery, David Beaune, Julie Boudreau, Marcella Chelli Nuzzo, Ruth Cox, Augusta Dorey, Ryan Gardiner, Guillermo Fandos Guzman, Rachael Forster, Abi Harrison, Karen Hornigold, Sam Jones, Martha Nash, Steven MacKenzie, Rebecca McGowan-Griffin, Eric McNeill, Luke Nichols, Charlotte Palmer, Sara Paul, Nick Pruijn, Sam Puls, Dario Rivera, Scott Schilds, Sarah Sells, Nina Storm, Sam Stubbs, Ben Wallis, Phil Wheeler, Geoff While, Clare Wilkinson, Katy Wilson, CB Woods and Amelia Zakiewicz. We acknowledge the Instituto de Conservación Forestal (ICF), who maintain the Base camp facilities. We are particularly grateful to Roberto Downing from Expediciones y Servicios Ambientales de Cusuco (ESAC) without whom fieldwork would not have been logistically possible. The primary author was supported by funding from the Department for Employment and Learning, Northern Ireland (http://www.delni.gov.uk). We are grateful to Dr Sarah Helyar for the use of her laboratory to conduct molecular genetic analysis for shrew identification. Thanks to Georg Hantke, Curatorial Preparator and Dr Andrew Kitchener, Principal Curator of Vertebrates in the Department of Natural Sciences for preparing and lodging Cryptosis shrew specimens in the National Museum of Scotland, Edinburgh, facilitating species identification. We thank Michael Cove, editor William Corrêa Tavares and another anonymous reviewer for their helpful feedback.

\section{Authors' contributions}

HH was the primary author, OB built and maintained the database of records, KD performed molecular genetic analyses on Crytotis spp, KS and NMcC led much of the data collection and coordination, MJ conceived of the study, FC led data collection for Panthera and NR was the primary supervisor. All authors were involved in data collection and read, edited and approved the manuscript.

\section{References}

Almendra AL, González-Cózatl F, Engstrom M, Rogers D (2018) Evolutionary relationships and climatic niche evolution in the genus Handleyomys (Sigmodontinae: Oryzomyini). Molecular Phylogenetic Evolution 128: 12-25. https://doi.org/10.1016/j.ympev. 2018.06.018

Baird AB, McCarthy TJ, Trujillo RG, Kang YY, Esmaeiliyan M, Valdez J, Woodman N, Bickham JW (2018) Molecular systematics and biodiversity of the Cryptotis mexicanus group (Eulipotyphla: Soricidae): two new species from Honduras supported. Systematics and Biodiversity 16 (2): 108-117. https://doi.org/10.1080/1477200 0.2017 .1333539

Bianchi R, Mendes S, Júnior P (2010) Food habits of the ocelot, Leopardus pardalis, in two areas in southeast Brazil. Studies on Neotropical Fauna and Environment 45 (3): 111-119. https://doi.org/10.1080/ 01650521.2010 .514791 .

BirdLife International (2017) The World Database of Key Biodiversity Areas. Developed by the Key Biodiversity Areas Partnership: BirdLife International, IUCN, Amphibian Survival Alliance, Conservation International, Critical Ecosystem Partnership Fund, Global Environment Facility, Global Wildlife Conservation, NatureServe, Royal Society for the Protection of Birds, World Wildlife Fund and Wildlife Conservation Society http://www.keybiodiversityareas. org. Accessed on: 2017-02-22.

Davis ML, Kelly MJ, Stauffer DF (2011) Carnivore co-existence and habitat use in the Mountain Pine Ridge Forest Reserve, Belize. Animal Conservation 14 (1): 56-65. https://doi.org/10.1111/j.14691795.2010.00389.x

Dillon A, Kelly MJ (2008) Ocelot home range, overlap and density: comparing radio telemetry with camera trapping. Journal of Zoology 275(4): 391-398.https://doi.org/10.1111/j.1469-7998.2008.00452.x

Cove MV, Spínola RM, Jackson VL, Sáenz JC, Chassot O (2013) Integrating occupancy modeling and camera-trap data to estimate medium and large mammal detection and richness in a Central American biological corridor. Tropical Conservation Science 6 (6): 781-795. https://doi.org/10.1177/194008291300600606

Foster P (2001) The potential negative impacts of global climate change on tropical montane cloud forests. Earth-Science Reviews 55 (1-2): 73-106. http://doi.org/10.1016/S0012-8252(01)00056-3

Foster RJ, Harmsen BJ, Doncaster CP (2010) Habitat use by sympatric jaguars and pumas across a gradient of human disturbance in Belize. Biotropica 42 (6): 724-731. http://doi.org/10.1111/j.17447429.2010.00641.x

Foster RJ, Harmsen BJ, Valdes B, Pomilla C, Doncaster CP (2010) Food habits of sympatric jaguars and pumas across a gradient of human disturbance. Journal of Zoology 280: 309-318. https://doi. org/10.1111/j.1469-7998.2009.00663.x

Fundación Ecologista (1994) Evaluación Ecológica Rápida (EER). Parque Nacional "El Cusuco" y Cordillera del Merendon. The Nature Conservancy and PACA (Proyecto Ambiental para Centro América), San Pedro Sula, Cortez, Honduras, 79 pp.

Garcìa M, Jordan C, O'Farril G, Poot C, Meyer N, Estrada N, Leonardo R, Naranjo E, Simons Á, Herrera A, Urgilés C, Schank C, Boshoff L, Ruiz-Galeano M (2016) Tapirus bairdii. The IUCN Red List of Threatened Species http://doi.org/10.2305/iucn.uk.2016-1.rlts. t21471a45173340.en. Accessed on: 2018-05-11.

Gonthier DJ, Castañeda FE (2013) Large- and medium-sized mammal survey using camera traps in the Sikre River in the Río Plátano Biosphere Reserve, Honduras. Tropical Conservation Science 6 
(4): 584-591. https://doi.org/10.1177/194008291300600409

Higuera D, Martín-López B, Sánchez-Jabba A (2012) Social preferences towards ecosystem services provided by cloud forests in the neotropics: implications for conservation strategies. Regional Environmental Change 13 (4): 861-872. https://doi.org/10.1007/ s10113-012-0379-1

ICF (2015) Anuario Estadístico Forestal de Honduras. Instituto Nacional de Conservación y Desarrollo forestal, Áreas Protegidas y Vida Silvestre Vol. 30. ICF, Tegucigalpa, 138pp.

ICF (1987) Decreto numero 87-87. http://icf.gob.hn/wp-content/ uploads/2015/09/AP-Decreto-87-87-Declaratoria-Bosques-Nublados.pdf. Accessed on: 2017-03-10.

Irwin DM, Kocher TD, Wilson AC (1991) Evolution of the cytochrome $b$ gene of mammals. Journal of Molecular Evolution 32: 128-144. http://doi.org/10.1007/BF02515385

Lenz BB, Jack KM, Spironello WR (2014) Edge effects in the primate community of the biological dynamics of Forest Fragments Project, Amazonas, Brazil. American Journal Physical Anthropology 155: 436-446. https://doi.org/10.1002/ajpa.22590

Le Saout S, Hoffmann M, Shi Y, Hughes A, Bernard C, Brooks TM, Bertzky B, Butchart SHM, Stuart SN, Badman T, Rodrigues ASL (2013) Protected areas and effective biodiversity conservation. Science 342 (6160): 803-805. http://doi.org/10.1126/science.1239268

Luna-Vega I, Morrone JJ, Alcántara Ayala O and Espinosa Organista D (2001) Biogeographical affinities among Neotropical cloud forests Plant Systematics and Evolution 228 (3-4): 229-239. http://doi. org/10.1007/s006060170031

McCann NP, Wheeler PM, Coles T and Bruford MW (2012) Rapid ongoing decline of Baird's tapir in Cusuco National Park, Honduras. Integrative Zoology 7 (4): 420-428. http://doi.org/10.1111/ j.1749-4877.2012.00312.x

McKinney T, Westin J, Serio-Silva J (2015) Anthropogenic habitat modification, tourist interactions and crop-raiding in howler monkeys. In: Kowalewski M, Garber P, Cortes-Ortiz L, Urbani B, Youlatos D (Eds) Howler Monkeys, Developments in Primatology: Progress and Prospects. Springer, New York, 281-311.

Mejía D (2001) Honduras. In: Kappelle, M, Brown A (Eds) Bosques Nublados del Neotrópico. INBio, Costa Rica, 242-282.

Moreno RS, Kays RW, Samudio R (2006) Competitive release in diets of Ocelot (Leopardus pardalis) and Puma (Puma concolor) after Jaguar (Panthera onca) Decline. Journal of Mammalogy 87 (4) 808-816. https://doi.org/10.1644/05-MAMM-A-360R2.1

Munari DP, Keller C, Venticinque EM (2011) An evaluation of field techniques for monitoring terrestrial mammal populations in Amazonia. Mammalian Biology 76 (4): 401-408. https://doi. org/10.1016/j.mambio.2011.02.007

Myers N, Mittermeier RA, Mittermeier CG, Fonseca GAB, Kent J (2000) Biodiversity hotspots for conservation priorities. Nature 403 (6772): 853-858. http://doi.org/10.1038/35002501

Niedballa, J, Courtiol A, Sollmann R (2017). camtrapR: Camera Trap Data Management and Preparation of Occupancy and Spatial Capture-Recapture Analyses. R package version 0.99.9. https:// CRAN.R-project.org/package $=$ camtrapR

Oksanen J, Blanchet FG, Friendly M, Kindt R, Legendre P, McGlinn D, Minchin PR, O'Hara RB, Simpson GL, Solymos P, Stevens MHH, Szoecs E, Wagner H (2018). vegan: Community Ecology Package. R package version 2.5-1. https:/CRAN.R-project.org/ package $=$ vegan

Ortiz-Lozada L, Pelayo-Martínez J, Mota-Vargas C, DemeneghiCalatayud A, Sosa V (2017). Absence of large and presence of medium-sized mammal species of conservation concern in a privately protected area of rain forest in southeastern Mexico. Tropical Conservation Science 10: 1-13. https://doi. org/10.1177/1940082917738093

Petracca LS, Frair JL, Cohen JB, Calderón AP, Carazo-Salazar J, Castañeda F, Corrales-Gutiérrez D, Foster RJ, Harmsen B, Hernández-Potosme S, Herrera L, Olmos M, Pereira S, Robinson HS, Robinson N, Salom-Pérez R, Urbina Y, Zeller KA, Quigley
H (2017) Robust inference on large-scale species habitat use with interview data: the status of Jaguars outside protected areas in Central America. Journal of Applied Ecology 55 (2) 1-12. https://doi. org/10.1111/1365-2664.12972

Ponce-Reyes R, Nicholson E, Baxter PWJ, Fuller RA, Possingham $\mathrm{H}$ (2013) Extinction risk in cloud forest fragments under climate change and habitat loss. Diversity and Distributions 19 (5-6): 518-529. https://doi.org/10.1111/ddi.12064

Quigley H, Foster R, Petracca L, Payan E, Salom R, Harmsen B (2017) Panthera onca. The IUCN Red List of Threatened Species 2017. http://doi.org/10.2305/iucn.uk.2017-3.rlts.t15953a50658693.en. Accessed on: 2018-05-11.

R Core Team (2017). R: A language and environment for statistical computing. R Foundation for Statistical Computing, Vienna, Austria. https://www.R-project.org/.

RStudio Team (2015). RStudio: Integrated Development for R. RStudio, Inc., Boston, MA. http://www.rstudio.com/.

Reid F (1997) A Field Guide to the Mammals of Central America and Southeast Mexico. 1st Edition. Oxford University Press, New York. 334 pp.

Reid F (2009) A Field Guide to the Mammals of Central America and Southeast Mexico. 2nd Edition. Oxford University Press, New York. 346 pp.

Rojas-Soto O, Sosa V, Ornelas J (2012) Forecasting cloud forest in eastern and southern Mexico: conservation insights under future climate change scenarios. Biodiversity and Conservation 21 (10): 2671-2690. http://doi.org/10.1007/s10531-012-0327-x

Ruedas LA, Salazar-Bravo J (2007) Morphological and chromosomal taxonomic assessment of Sylvilagus brasiliensis gabbi (Leporidae). Mammalia 71 (1-2): 63-69. http://doi.org/10.1515/ MAMM.2007.011

Scatena FN, Bruijnzeel LA, Bubb P, Das S (2010) Setting the stage. In: Bruijnzeel LA, Scatena FN, Hamilton LS (Eds) Tropical Montane Cloud Forests: Science for Conservation and Management. Cambridge University Press, Cambridge, 3-13.

Schank CJ, Cove MV, Kelly MJ, Mendoza E, O'Farrill G, ReynaHurtado R, Jordan CA, González-Maya JF, Lizcano DJ, Moreno R, Dobbins MT, Montalvo V, Sáenz-Bolaños C, Carillo Jimenez E, Estrada N, Cruz Díaz JC, Saenz J, Spínola M, Carver A, Fort J, Nielsen CK, Botello F, Pozo Montuy G, Rivero M, de la Torre JA, Brenes-Mora E, Godínez-Gómez O, Wood MA, Gilbert J, Miller JA (2017) Using a novel model approach to assess the distribution and conservation status of the endangered Baird's tapir. Diversity and Distributions 23 (12): 1459-1471. https://doi.org/10.1111/ ddi. 12631

Smith MF, Patton JL (1993) Diversification of South American murid rodents: evidence from mitochondrial DNA sequence data for the akodontine tribe. Biological Journal of the Linnean Society 50: 149-177. https://doi.org/10.1111/j.1095-8312.1993.tb00924.x

Stadtmüller T (1987) Cloud Forests in the Humid Tropics: A Bibliographic Review. The United Nations University Press, Tokyo, 82 pp.

Sunquist M, Sunquist F (2002) Wild Cats of the World. University of Chicago Press, Chicago and London, 452 pp.

Thornton D, Branch L, Sunquist, M (2011) Passive sampling effects and landscape location alter associations between species traits and response to fragmentation. Ecological Applications 21 (3): 817-829.

Tobler MW, Carrillo-Percastegui SE, Leite Pitman R, Mares R, Powell G (2008). An evaluation of camera traps for inventorying large-and medium-sized terrestrial rainforest mammals. Animal Conservation 11 (3): 169-178. https://doi.org/10.1111/j.14691795.2008.00169.x

Wickham H (2009) ggplot2: Elegant Graphics for Data Analysis. Springer-Verlag, New York.

Wilson DE, Lacher TE Jr, Mittermeier RA (2017) Handbook of the Mammals of the World. Rodents II. Lynx Editions, Barcelona, $1008 \mathrm{pp}$.

Woodman N (2011) Nomenclatural notes and identification of small- 
eared shrews (Mammalia: genus Cryptotis) from Cobán, Guatemala, in the Natural History Museum, London. Proceedings of the Biological Society of Washington 124: 249-258. https://doi. org/10.2988/11-11.1

Woodman N (2015) Morphological variation among broad-clawed shrews (Mammalia: Eulipotyphla: Soricidae: Cryptotis Pomel, 1848) from highlands of Western Honduras, with descriptions of three new cryptic species. Annals of Carnegie Museum 83 (2): 95-119. https://doi.org/10.2992/007.083.0203

Wultsch C, Caragiulo A, Dias-Freedman I, Quigley H, Rabinowitz S, Amato G (2016) Genetic diversity and population structure of mesoamerican Jaguars (Panthera onca): implications for conservation and management. PLoS ONE 11 (10): e0162377. https://doi. org/10.1371/journal.pone.0162377

\section{Appendix}

\begin{tabular}{|c|c|c|c|c|c|c|c|c|c|c|c|}
\hline \multicolumn{12}{|c|}{ Camps surveyed for small mammals } \\
\hline & 2006 & 2007 & 2008 & 2009 & 2010 & 2011 & 2012 & 2013 & 2014 & 2015 & 2016 \\
\hline Buenos Aires & $\mathrm{Y}$ & Y & $\mathrm{Y}$ & $\mathrm{Y}$ & $\mathrm{Y}$ & $\mathrm{N}$ & $\mathrm{N}$ & $\mathrm{Y}$ & $\mathrm{Y}$ & $\mathrm{N}$ & $\mathrm{N}$ \\
\hline Base camp & Y & $\mathrm{Y}$ & Y & $\mathrm{Y}$ & $\mathrm{Y}$ & $\mathrm{N}$ & $\mathrm{Y}$ & $\mathrm{Y}$ & $\mathrm{Y}$ & $\mathrm{Y}$ & $\mathrm{Y}$ \\
\hline Cantiles & $\mathrm{Y}$ & Y & $\mathrm{Y}$ & $\mathrm{Y}$ & $\mathrm{N}$ & $\mathrm{N}$ & $\mathrm{N}$ & $\mathrm{Y}$ & $\mathrm{Y}$ & $\mathrm{Y}$ & Y \\
\hline Cortecito & $Y$ & Y & Y & $\mathrm{N}$ & $\mathrm{N}$ & $\mathrm{N}$ & $Y$ & $Y$ & $Y$ & Y & $Y$ \\
\hline Danto & $Y$ & $Y$ & $Y$ & $\mathrm{~N}$ & $\mathrm{~N}$ & $\mathrm{~N}$ & $\mathrm{~N}$ & $Y$ & $Y$ & $\mathrm{Y}$ & $Y$ \\
\hline Guanales & $\mathrm{Y}$ & $\mathrm{Y}$ & Y & $\mathrm{N}$ & $\mathrm{N}$ & $\mathrm{N}$ & $\mathrm{N}$ & $\mathrm{Y}$ & $Y$ & $\mathrm{Y}$ & $\mathrm{Y}$ \\
\hline Santo Tomas & $\mathrm{N}$ & $\mathrm{Y}$ & $\mathrm{Y}$ & $\mathrm{N}$ & $\mathrm{N}$ & $\mathrm{N}$ & $\mathrm{N}$ & $\mathrm{Y}$ & $Y$ & Y & $\mathrm{N}$ \\
\hline \multicolumn{12}{|c|}{ Total line transect km per camp each year } \\
\hline & 2006 & 2007 & 2008 & 2009 & 2010 & 2011 & 2012 & 2013 & 2014 & 2015 & 2016 \\
\hline Base camp & 8.5 & 0 & 16.9 & 20 & 12.7 & 14.8 & 14.8 & 14.8 & 7.4 & 9.2 & 7.4 \\
\hline Buenos Aires & 0 & 0 & 12.88 & 5.94 & 11.88 & 11.88 & 11.88 & 5.94 & 5.94 & 0 & 0 \\
\hline Cantiles & 18.7 & 7.4 & 21.1 & 14 & 13.2 & 13.2 & 13.2 & 6.6 & 6.6 & 6.6 & 6.6 \\
\hline Cortecito & 35 & 22.3 & 21.4 & 8.7 & 17.4 & 17.4 & 16.4 & 8.7 & 7.7 & 6.7 & 6.7 \\
\hline Danto & 23.4 & 17.7 & 18.6 & 15.2 & 18.6 & 13.4 & 15 & 9.3 & 14.6 & 7.3 & 7.3 \\
\hline Guanales & 22.79 & 13.03 & 13.46 & 10.43 & 14.09 & 13.46 & 13.46 & 6.73 & 6.73 & 6.73 & 6.73 \\
\hline Santo Tomas & 0 & 0 & 8.74 & 4.37 & 5.94 & 8.37 & 7.64 & 4.37 & 0 & 0 & 0 \\
\hline \multicolumn{12}{|c|}{ Camera trapping placement per camp } \\
\hline & & & & & & & & & 2014 & 2015 & 2016 \\
\hline Base Camp & & & & & & & & & 8 & 24 & 20 \\
\hline Buenos Aires & & & & & & & & & 4 & 0 & 0 \\
\hline Cantiles & & & & & & & & & 5 & 23 & 21 \\
\hline Cortecito & & & & & & & & & 6 & 16 & 17 \\
\hline Danto & & & & & & & & & 11 & 22 & 18 \\
\hline Guanales & & & & & & & & & 8 & 20 & 18 \\
\hline
\end{tabular}

